\title{
Scaling Equations from a Self-Consistent Theory of Anderson Localization
}

\author{
D. Vollhardt \\ Max-Planck-Institut für Physik und Astrophysik, D-8000 Munich 40, Federal Republic of Germany \\ and \\ P. Wölfle \\ Physik-Department der Technischen Universität München, D-8046 Garching, Federal Republic of Germany, and \\ Max-Planck-Institut für Physik und Astrophysik, D-8000 Munich 40, Federal Republic of Germany \\ (Received 6 November 1981) \\ The conductance of a disordered system of finite volume $L^{d}$ ( $d$ spatial dimensions) is \\ calculated by making use of a recently developed self-consistent theory. Scaling equa- \\ tions are derived for the dimensionless conductance $g$ and the scaling function $\beta(g(L))$ \\ $=d \ln g / d \ln L$ is explicitly calculated for arbitrary dimension $d$. It is shown that the \\ theory obeys scaling in the sense of Wegner, Thouless, and Abrahams et al. \\ PACS numbers: $72.10 . \mathrm{Bg}, 71.30 .+\mathrm{h}$
}

Some time ago Abrahams et al., ${ }^{1}$ using ideas developed earlier by Thouless, ${ }^{2}$ proposed a scaling theory of the Anderson transition in which the dimensionless conductance $g$ as a function of a sample length $L$ was assumed to be the only relevant scaling parameter. In particular, the limiting behavior of the scaling function $\beta(g)$ was found to $\mathrm{be}^{1,3}$

$$
\beta(g)=\frac{d \ln g}{d \ln L}=d-2-\frac{\alpha}{g}+O\left(\frac{1}{g^{2}}\right) .
$$

Earlier Wegner ${ }^{4}$ had proposed a mapping of the Anderson tight-binding Hamiltonian onto nonlinear $\sigma$ models, for which the equivalent of the conductance obeys similar scaling equations. Recently other field-theoretical calculations employed similar models to calculate the $\beta$ function perturbationally to higher order in $1 / g^{5,6}$

Starting from a systematic investigation of infrared divergences in the extended-state perturbation theory, we have recently derived a self consistent diagrammatic theory of the density response function. ${ }^{7,8}$ The idea of a self-consis tent formulation of the density response was introduced earlier into this field in combination with a mode-coupling theory by Götze. ${ }^{9,10}$ Our treatment $^{7}$ yields a lower critical dimension $d=2$, the results for the dielectric constant and the frequency -dependent conductivity in $d=1$ being in good agreement with exact solutions for the weak-coupling case.

In this communication we want to demonstrate that the self-consistent theory may also be used to calculate other quantities, 'e.g., the (lengthdependent) conductance of a finite system of length $L$.

For small frequency $\omega$ and wave vector $q$ the density response function may be expressed in terms of the diffusion coefficient $D(q, \omega)$ [Eq. (30) in Ref. 7]. We have previously derived a selfconsistent equation for $D(q=0, \omega) \equiv D(\omega)$ by a diagrammatic method (the $q$ dependence of $D$ will be addressed in the context of dimensions $d>2$ ), which may be written as ${ }^{7}$

$$
D(\omega)=D_{0}-\frac{k_{\mathrm{F}}^{2-d}}{\pi m} \int_{0}^{k_{0}} d k \frac{k^{d-1}}{[-i \omega / L(\omega)]+k^{2}} .
$$

Here $D_{0}$, the bare diffusion constant, is given by $D_{0}=(\pi d m \lambda)^{-1}$, where $\lambda$, the disorder parameter, may be expressed in the weak-coupling limit by the transport relaxation time as $\lambda$ $=\left(2 \pi E_{\mathrm{F}} \tau\right)^{-1}$. Equation (2) essentially is an integral over a density relaxation function, ${ }^{11}$ which has a diffusion pole structure at least in the hy drodynamic regime $k<1 / l$, where $l$ is the mean free path. It will later become evident, however, that the contribution from higher momenta is negligible for the calculation of critical properties.

For small frequencies $\omega$ the conductivity $\sigma(\omega)$ is given by $\sigma(\omega)=\sigma_{0} D(\omega) / D_{0}$, where $\sigma_{0}=e^{2}(n / m) \tau$ ( $n$, density; $m$, mass of electrons). In the insulating state the polarizability $\alpha(\omega)$ defined by $\sigma(\omega)$ $=-i \omega \alpha(\omega)$ is finite in the limit $\omega \rightarrow 0$ and hence we expect $D(\omega) \propto-i \omega$. The density response function assumes a form describing exponential localization, ${ }^{9}$ i.e.,

$$
\lim _{\omega \rightarrow 0} \chi(q, \omega)=\left[q^{2} \xi^{2} /\left(1+q^{2} \xi^{2}\right)\right] \chi_{T}(q, 0),
$$

where $\xi=\lim _{\omega \rightarrow 0}[-i \omega / D(\omega)]^{-1 / 2}$ is the localization length. Taking the limit $\omega \rightarrow 0$ in (2) we derive the following implicit equation for the local- 
ization length:

$$
1=\lambda d\left(k_{\mathrm{F}} \xi\right)^{2-d} \int_{0}^{k_{0} \xi} d y \frac{y^{d-1}}{1+y^{2}} .
$$

Equations of the form of (2) and (3) have essentially been derived in Refs. 9 and 10 within a mode-coupling theory the difference, however, being an additional factor $\left(k / k_{\mathrm{F}}\right)^{2}$ in the integrand. Generalizing the mode-coupling theory such that the exact crossing symmetry between the particle-particle $(p-p)$ and the particle-hole $(p-h)$ channel in the case of time-reversal invariance is satisfield, Prelovsek ${ }^{12}$ obtained equations identical to (2) and (3) for small $\omega$ and $q$.

In our previous work ${ }^{7}$ we had treated the problem only for $d \leqslant 2$ dimensions, since in this case the perturbation series contains infrared divergences for arbitrarily small coupling constant $\lambda$. In higher dimensions the diffusion pole $\left(\omega+i D k^{2}\right)^{-1}$ is removed by the Jacobian factor $k^{d-1}$. However, as one approaches the Anderson transition by increasing the coupling strength, the diffusion coefficient tends to zero. In this case the diffusion propagator diverges in the limit $\omega \rightarrow 0$ even for finite momentum $k$. Thus the same terms in the perturbation series which give rise to infrared singularities for $d \leqslant 2$ will now also diverge. One therefore finds that a diverging contribution is once more provided by the diffusion pole in the $\mathrm{p}-\mathrm{p}$ channel ( $2 k_{\mathrm{F}}$ singularity), which is due to the crossing symmetry of the four-point function in the case of time-reversal invariance. In general there is also a contribution from the diffusion pole in the $\mathrm{p}-\mathrm{h}$ channel which we have identified diagrammatically in Fig. 4 of Ref. 7. In that paper we proved that the divergent terms in the perturbation series arising in $d \leqslant 2$ from this set of diagrams are cancelled in the weak-coupling limit. More precisely, writing the divergent expression due to the $\mathrm{p}-\mathrm{h}$ diffusion poles as $\sum_{\mathrm{k}} Z(\overrightarrow{\mathrm{k}}) /\left(-i \omega+D k^{2}\right)$ this cancellation implies $\lim _{|\overrightarrow{\mathrm{k}}| \rightarrow 0} Z(\overrightarrow{\mathrm{k}})=0$. Nonetheless, for $D$ going to zero (as one approaches the Anderson transition) this expression will allow for a divergence for nonzero $k$ values. Götze ${ }^{9,10}$ has shown that this divergence gives rise to a metal-insulator transition and has given a detailed discussion of the corresponding self-consistent mode-coupling theory. Most recently both mechanisms have been investigated simultaneously within the modecoupling theory. ${ }^{13}$ The results indicate ${ }^{13}$ that for short-range potentials the $\mathrm{p}-\mathrm{h}$ and $\mathrm{p}-\mathrm{p}$ diffusion poles give comparable contributions. However, the dynamics near the Anderson transition is found $^{13}$ to be governed by the $p-p$ diffusion pole (i.e., quantum interference effects). We have independently reached the latter conclusion within our diagrammatic treatment by extending (2) to contain also the contribution from $\mathrm{p}$-h diffusion poles. ${ }^{7}$ As we are indeed interested in the scaling properties near the Anderson transition, we believe it sufficient to keep only the $p-p$ diffusion contribution. Consequently we are again led to the self-consistent equation (2) for the diffusion coefficient $D .^{14}$

Rewriting (2) and performing the integral we obtain

$$
\frac{D(\omega)}{D_{0}}=1-\frac{d \lambda}{d-2}\left(\frac{k_{0}}{k_{\mathrm{F}}}\right)^{d-2}+d \lambda\left(\frac{-i \omega}{D{k_{\mathrm{F}}}^{2}}\right)^{(d-2) / 2} \int_{0}^{b(\omega)} d x \frac{x^{d-3}}{1+x^{2}},
$$

where $b(\omega) \equiv\left[i D(\omega) k_{0}{ }^{2} / \omega\right]^{1 / 2}$. This expression defines the critical coupling strength $\lambda_{c}=\lfloor(d-2) /$ $d]\left(k_{0} / k_{\mathrm{F}}\right)^{2-d}$, such that $D(0) / D_{0}=1-\lambda / \lambda_{c}$ for $\lambda$ $<\lambda_{c}$. Equation (4) can be discussed for $\lambda \lessgtr \lambda_{c}$ and allows one to determine the critical exponents $s$ of the dc conductivity $\sigma(0)$ (namely $s=1$ for all $d$ ) and $\nu$ of the localization length $\xi$ for arbitrary dimension $d$ by explicit calculation of $\sigma(0)$ and $\xi .^{11}$ For $2<d<4$ one obtains $\nu=1 /(d-2)$, i.e., the results obey the scaling law $s=(d-2) \nu$ first derived by Wegner ${ }^{15}$; for $d>4$ we get $\nu=\frac{1}{2}$. These values of $s$ and $\nu$ have been found earlier in Ref. 12. In $d=4$, however, we obtain ${ }^{11}$ logarithmic corrections to $\nu=\frac{1}{2}$, in contrast to Ref. 12 .

For $b(\omega) \gg 1$, which always holds for small enough $\omega$ near the transition, ${ }^{16}$ the upper limit in the integral of (4) can be extended to infinity. For $2<d<4$ one therefore obtains ${ }^{16}$

$$
\frac{D(\omega)}{D_{0}}=1-\frac{\lambda}{\lambda_{c}}+p \frac{\lambda}{\lambda_{c}}\left[\frac{-i \omega}{D(\omega) k_{0}^{2}}\right]^{(d-2) / 2},
$$

where $p=\Gamma^{\prime}(d / 2) \Gamma(2-d / 2)$. An essentially identical equation has recently been derived by Hikami ${ }^{6}$ using a field-theoretical formalism (nonlinear $\sigma$ model) and employing the renormalization-group method. Indeed it can be shown directly ${ }^{16}$ that the frequency-dependent conductivity $\sigma(\omega) \propto D(\omega)$ as determined by the self-consistent equation (4), close to the Anderson transition, has the scaling form $\sigma(\omega) \propto \omega^{(d-2) / d} F\left(\omega / \omega_{c}\right)$ as proposed by scaling theory ${ }^{17}$ where $\omega_{c}$ is a crossover frequency, 
$\omega_{c} \propto\left|1-\lambda / \lambda_{c}\right|^{d /(d-2)}$. Within a self-consistent formulation a scaling relation of this form was first derived in Ref. 9 for $d=3$, where, however, $\omega_{c} \propto\left|1-\lambda / \lambda_{c}\right|^{3 / 2}$.

We now discuss the case of finite samples. The mechanism causing localization, i.e., the quantum interference of infinitely many successive impurity scattering processes, is still working in this case. However, the electron now has a finite probability to reach the end of the sample before it eventually turns back. This effect is expressed by restricting the available diffusionmode wave vector to $k>1 / L$. We may therefore calculate the length-dependent diffusion coefficient from (2), which is a momentum integral over a correlation function describing the spatial decay of density fluctuations, by introducing a lower cutoff at $k=1 / L$. In the limit $\omega \rightarrow 0$ the characteristic length is defined by $\xi=\lim _{\omega \rightarrow 0}(-i \omega /$ $D)^{-1 / 2}$, the localization length. This length only depends on microscopic quantities and, in particular, not on the length of the sample. We therefore replace $-i \omega / D$ by $\xi^{-2}$ in the denominator of the integral in (2), i.e., its value in the infinite system. This is consistent with renormalization-group treatments ${ }^{5,6}$ where $-i \omega / D$ is found to be independent of $L$. One therefore obtains

$$
\frac{D(L)}{D_{0}}=1-d \lambda \xi^{2} k_{\mathrm{F}}^{2-d} \int_{1 / L}^{k_{0}} d k \frac{k^{d-1}}{1+k^{2} \xi^{2}} .
$$

We now proceed to calculate the dimensionless conductance $g$, which according to Abrahams et $a l .{ }^{1}$ is the quantity obeying scaling. A scalar potential $U(x)=e E(L-x)$, corresponding to an electric field $E$, gives rise to a change in density $\delta \rho(x)$, which in turn drives a diffusion current. At the end of the sample $(x=L)$ the current den- sity is given by $J=e D(L)[d(\delta \mu) / d x]_{x=L}$ which must be equal and opposite to the electric cur rent. In calculating $\delta \rho$ from the applied potential it is essential that the density response in the localized regime is nonlocal, and

$$
J=e D(L)\left[(d / d x) \int_{0}^{L} d x^{\prime} \chi\left(x-x^{\prime}\right) U\left(x^{\prime}\right)\right]_{x=L} \text {. }
$$

By Fourier transforming $\chi(q, 0)$ one finds

$$
\chi\left(x-x^{\prime}\right)=N_{\mathrm{F}}\left[\delta\left(x-x^{\prime}\right)-(2 \xi)^{-1} \exp \left(-\left|x-x^{\prime}\right| / \xi\right)\right]
$$

where $N_{\mathrm{F}}=\left[S_{d} /(2 \pi)^{d}\right] m k_{\mathrm{F}}{ }^{d-2}$ and $S_{d}$ is the surface of the $d$-dimensional unit sphere. Substituting this into the expression for $J$ and subtracting (6) and (3), the dimensionless conductance $g(L)=\left(e^{2} /\right.$ $\hbar)^{-1} L^{d-2} J / E$ is finally obtained as

$$
g(L)=c_{d} x^{d-2}(1+x) e^{-x} \int_{0}^{1 / x} d y y^{d-1} /\left(1+y^{2}\right),
$$

where we defined $x=L / \xi$ and $c_{d}=(2 / \pi)\left[S_{d} /(2 \pi)^{d}\right]{ }^{15}$ One observes that $g(L)$ only depends on a single parameter, namely the scaled length of the sample; e.g., for $d=2$ one obtains

$$
g(L)=\left(2 \pi^{2}\right)^{-1} \ln \left[1+(\xi / L)^{2}\right](1+L / \xi) e^{-L / \xi} .
$$

For $\lambda<\lambda_{c}$ (and $d>2$ ) we find

$$
g(L)=c_{d}(d-2)^{-1}\left[1+\Gamma(d / 2) \Gamma(2-d / 2)(L / \xi)^{d-2}\right]
$$

where the characteristic length $\xi$ is defined ${ }^{11}$ in analogy to the localization length, i.e., $\xi=k_{0}{ }^{-1}(1$ $\left.-\lambda / \lambda_{c}\right)^{-1 /(d-2)}$.

Clearly, as $g$ is a function of the single variable $L / \xi$ there exists a single-parameter scaling equation. We may calculate the scaling function $\beta(g(L))=d \ln g / d \ln L$ explicitly from (7):

$$
\beta(g(L))=d-2-\frac{c_{d}}{g} \frac{(1+x) e^{-x}}{1+x^{2}}-\frac{x^{2}}{1+x},
$$

where $x=L(g) / \xi$ and $L(g)$ is the inverse function of $g(L)$, i.e., $\beta$ is a function of $g$ only. The most interesting dimension is $d=2$, where

$$
\beta(g)=\left\{\begin{array}{l}
-\left(\pi^{2} g\right)^{-1}-\left\lfloor 1-3 /\left(2 \pi^{2} g\right)\right] \exp \left(-2 \pi^{2} g\right)+O\left(\exp \left(-3 \pi^{2} g\right)\right), \quad g \gg 1, \\
\ln g+O\left(\ln \left(\ln g^{-1}\right)\right), \quad g \ll 1 .
\end{array}\right.
$$

It is interesting to note that the scaling function in $d=2$ is nonanalytic for $g \rightarrow \infty$ (nonperturbative contribution to $\beta$ ). Hence in a formal expansion of $\beta(g)$ in powers of $1 / g$ the coefficients of $(1 / g)^{n}$, $n \geqslant 2$, are zero. This is consistent with the finding in Refs. 5 and 6 , where it was shown that the coefficient of the $1 / g^{2}$ term ${ }^{5}$ and even of the $1 / g^{3}$ term $\mathrm{m}^{6}$ vanishes. For $\lambda<\lambda_{c}$ one finds $\beta(g)=d-2$ $-c_{d} / g$ from the expression for $g(L)$ below (8). In Fig. 1 we have plotted $\beta(g)$ vs $\ln g$ for dimensions $d=1,2,3$.
It is also instructive to calculate $\beta(g)$ for infinite sample length $L$ but finite frequency $\omega$, i.e., $\beta(g(\omega))=d \ln g / d \ln \omega$ where ${ }^{11}$ the scaling parameter is

$$
g(\omega)=\left(e^{2} / \hbar\right)^{-1} \xi^{d-2} \sigma(\omega)\left(-i \omega / \omega_{c}\right)^{(2-d) / d}
$$

and $\omega_{c} \propto \xi^{-d}$. For $d=2$ one obtains $\beta(g(\omega))=(1$ $\left.+2 \pi^{2} g\right)^{-1}$. We see that $\beta(g(L))$ and $\beta(g(\omega))$ only agree to lowest order $\left(\omega \propto L^{-2}\right)$, i.e., for $g \rightarrow \infty$. Observe that the $\beta$ function calculated in renor- 


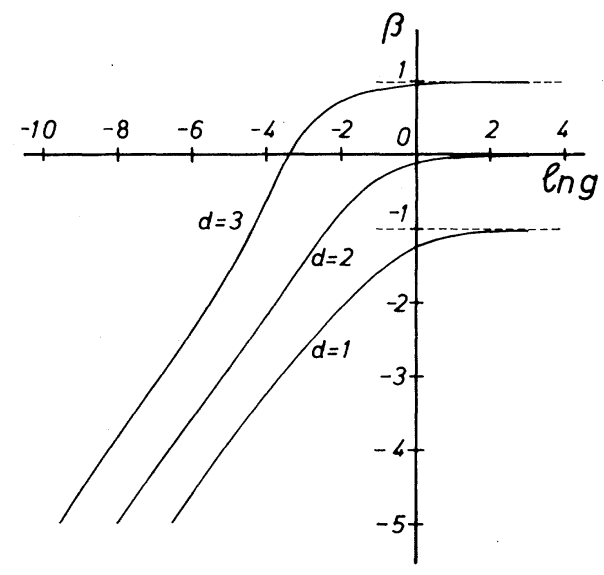

FIG. 1. The scaling function $\beta(g(L))=d \ln g / d \ln L$ vs $\ln g$ is shown for dimensions $d=1,2,3$.

malization-group treatments ${ }^{4-6}$ corresponds to $^{-}$ $\beta(g(L))$ rather than $\beta(g(\omega))$. From (9) one can easily find the critical conductance $g_{c}$ at which $\beta$ vanishes for $d>2$ (i.e., at the Anderson transition, where $\xi \rightarrow \infty)$. It is given by $g_{c}=c_{d} /(d-2)$. The slope of $\beta(g(L))$ at $g_{c}$ is therefore found to be $[d \beta / d \ln g]_{B_{c}}=\nu^{-1}$.

In conclusion, we have shown that a self-consistent treatment of the Anderson localization problem yields a dimensionless conductance $g$ which is a function of the reduced length variable $L / \xi$ only. Here $g$ obeys a scaling equation as proposed by Abrahams et al.$^{1}$ for all dimensions $d$. The scaling function $\beta(g)$ can be evaluated explicitly. Our results from the self-consistent theory are in agreement with exact results of field-theoretical models that are thought to describe the same system and which assume scaling.

We would like to thank Professor W. Götze for useful comments and for informing us about his recent work. One of us (D.V.) is grateful for the hospitality extended to him by the Aspen Center for Physics during the workshop on Localization and Interactions in Impure Metals where the results of this paper were first discussed. He thanks the participants of the workshop for numerous, valuable discussions.

${ }^{1}$ E. Abrahams, P. W. Anderson, D. C. Licciardello, and T. V. Ramakrishnan, Phys. Rev. Lett. $\underline{42}, 672$ (1979).

${ }^{2}$ D. J. Thouless, Phys. Rev. Lett. 39, 1167 (1977).

${ }^{3}$ L. P. Gorkov, A. I. Larkin, and D. E. Khmel'nitskii, Pis'ma Zh. Eksp. Teor. Fiz. 30, 248 (1979) [JETP Lett. 30, 228 (1979)].

${ }^{4}$ F. J. Wegner, Z. Phys. B 35, 207 (1979).

${ }^{5}$ K. B. Efetov, A. I. Larkin, and D. E. Khmel'nitskii, Zh. Eksp. Teor. Fiz. 79, 1120 (1980) [Sov. Phys. JETP $42,568(1980)]$.

${ }^{6} \mathrm{~S}$. Hikami, private communication.

${ }^{7}$ D. Vollhardt and P. Wölfle, Phys. Rev. Lett. $\underline{45}, 842$ (1980), and Phys. Rev. B 22, 4666 (1980).

${ }^{8} \mathrm{~A}$ diagrammatic, self-consistent theory has also been considered more recently by A. Kawabata, Solid State Commun. 38, 823 (1981).

${ }^{9}$ W. Götze, Solid State Commun. 27, 1393 (1978), and J. Phys. C 12, 1279 (1979).

${ }^{10}$ W. Götze, Philos. Mag. B 43,219 (1981).

${ }^{11} \mathrm{P}$. Wölfle and D. Vollhardt [to be published in Springer Series in Solid State Sciences (Springer-Verlag, Berlin)].

${ }^{12}$ P. Prelovsek, Phys. Rev. B 23, 1304 (1981), and in Recent Developments in Condensed Matter Physics, edited by J. T. Devreese (Plenum, New York, 1981), Vol. II, p. 191.

${ }^{13}$ D. Belitz, A. Gold, and W. Götze, Z. Phys. B 44 , 273 (1981).

${ }^{14}$ From a purely diagrammatic point of view, using the maximally crossed diagrams (Refs. 1 and 3 ) to calculate corrections to the diffusion constant $D_{0}$, there is no significant $q$ dependence of $D$. However, scaling theory suggests that $D$ has a $D(q, \omega) \propto q^{d-2}$ dependence close to the mobility edge. (E. Abrahams, P. W. Anderson, and P. A. Lee, private communication.) Although one does not expect the momentum dependence to be important for the calculation of $D(0, \omega)$, the implications of this fact for a self-consistent theory are not yet understood.

${ }^{15}$ F. J. Wegner, Z. Phys. B 25, 327 (1976).

${ }^{16} \mathrm{~B}$. Shapiro, to be published.

${ }^{17}$ S. Hikami, Phys. Rev. B 24, 2671 (1981); B. Shapiro and E. Abrahams, Phys. Rev. B 24, 4889 (1981). 